\title{
En bloc esophagectomy reduces local recurrence and improves survival compared with transhiatal resection after neoadjuvant therapy for esophageal adenocarcinoma
}

\author{
C. Rizzetto, MD, ${ }^{a}$ S. R. DeMeester, MD, ${ }^{\text {b }}$ J. A. Hagen, MD, ${ }^{a}$ C. G. Peyre, MD, ${ }^{a}$ J. C. Lipham, MD, ${ }^{a}$ and T. R. DeMeester, MD $^{a}$
}

Earn CME credits at http:// cme.ctsnetjournals.org
From the Departments of Surgery ${ }^{\text {a }}$ Cardiothoracic Surgery, ${ }^{\mathrm{b}}$ the Keck School of Medicine, University of Southern California, Los Angeles, Calif.

Read at the Thirty-third Annual Meeting of the Western Thoracic Surgical Association, Sanata Ana Pueblo, NM, June 27-30, 2007.

Received for publication July 2, 2007; revisions received Sept 15, 2007; accepted for publication Oct 4, 2007.

Address for reprints: Steven R. DeMeester, MD, Department of Cardiothoracic Surgery, University of Southern California, 1510 San Pablo St, Suite 514, Los Angeles, CA 90033 (E-mail: sdemeester@surgery.usc.edu).

J Thorac Cardiovasc Surg 2008;135:122836

\section{$0022-5223 / \$ 34.00$}

Copyright $(0) 2008$ by The American Association for Thoracic Surgery

doi:10.1016/j.jtcvs.2007.10.082
Objective: Neoadjuvant therapy is commonly used for esophageal adenocarcinoma. We have reported reduced local recurrence rates and improved survival after an en bloc esophagectomy compared with a transhiatal resection as primary therapy for adenocarcinoma of the esophagus. The aim of this study was to determine whether the benefits of an en bloc resection would extend to patients after neoadjuvant therapy.

Methods: The charts of all patients with esophageal adenocarcinoma that had neoadjuvant therapy and en bloc or transhiatal esophagectomy from 1992-2005 were reviewed. Patients found to have systemic metastatic disease at the time of the operation or who had an incomplete resection were excluded.

Results: There were 58 patients: 40 had an en bloc resection and 18 had a transhiatal esophagectomy. A complete pathologic response occurred in 17 (29.3\%) of 58 patients. Median follow-up was 34.1 months after en bloc resection and 18.3 months after transhiatal resection $(P=.18)$. Overall survival at 5 years and survival in patients with residual disease after neoadjuvant therapy was significantly better with an en bloc resection (overall survival: $51 \%$ for en bloc resection and $22 \%$ for transhiatal resection $[P=.04]$; survival with residual disease: $48 \%$ for en bloc resection and $9 \%$ for transhiatal resection $[P=.02])$. Survival in patients with complete pathologic response tended to be better after an en bloc resection (en bloc, $70 \%$; transhiatal, $43 \% ; P=.3$ ).

Conclusion: An en bloc resection provides a survival advantage to patients after neoadjuvant therapy compared with a transhiatal resection, particularly for those with residual disease. Similar to patients treated with primary resection, an en bloc esophagectomy is the procedure of choice after neoadjuvant therapy.

$\mathrm{T}$ The incidence of adenocarcinoma of the esophagus and esophagogastric junction is increasing faster than any other cancer in the Western world. ${ }^{1}$ Although survival is excellent in patients with early-stage tumors, unfortunately, most patients present with dysphagia and have locally advanced disease. The prognosis for these patients is poor, and consequently, attention has focused on the use of neoadjuvant therapy in an effort to reduce the local-regional tumor burden and eradicate micrometastatic systemic disease. During the past 2 decades, neoadjuvant chemoradiotherapy has been tested in numerous trials, and although a clear improvement in overall survival compared with that after surgical resection alone has not been demonstrated, subgroup analysis has suggested that patients who obtain a complete pathologic response have improved survival compared with those who have residual disease at the time of resection. ${ }^{2}$ This finding has prompted some oncologists to recommend eliminating surgical resection after neoadjuvant therapy because it is thought that those with complete pathologic response do not need resection and those with incomplete response have such a poor prognosis that they are unlikely to benefit from the operation. 


\section{Abbreviations and Acronyms \\ $\mathrm{CT}=$ computed tomography \\ $\mathrm{PET}=$ positron emission tomography}

It has been our policy to reserve neoadjuvant therapy for patients with locally advanced disease based on findings from computed tomographic (CT) scans, endoscopic ultrasonographic analysis, and/or positron emission tomographic (PET) scanning. After neoadjuvant therapy, we have taken fit patients for an en bloc resection, given evidence that local recurrence is minimized and survival is maximized with this procedure in patients who have surgical intervention as the primary therapy for their cancer. ${ }^{3}$ The aim of this study was to review our experience and determine whether the local control and survival benefits of an en bloc esophagectomy would extend to patients after neoadjuvant therapy and in particular to those with residual disease on final pathology.

\section{Materials and Methods Patient Population}

The study population was drawn from all patients who had an esophagectomy for adenocarcinoma between 1993 and 2005 at the University of Southern California. Assessment of the extent of disease before surgical intervention included upper endoscopy, a chest and abdominal CT scan, and, when it became available, endoscopic ultrasonographic analysis (1997) and PET or CT-PET scanning (1998). Patients were considered to have advanced local-regional disease when there was evidence of multiple $(>4)$ enlarged lymph nodes based on endoscopic ultrasonographic analysis. In patients who did not (unavailable) or could not (because of the size of the tumor) undergo endoscopic ultrasonography, a diagnosis of advanced local-regional disease was based on the endoscopic size and length of the tumor, CT scan evidence of a bulky tumor with or without enlarged peritumoral nodes, and/or PET scan findings consistent with multiple involved lymph nodes.

The records of all patients who had neoadjuvant therapy followed by an en bloc or a transhiatal esophagectomy for esophageal adenocarcinoma were reviewed. Neoadjuvant therapy was chemotherapy alone or chemoradiotherapy. Chemotherapy consisted of standard agents, including 5-fluorouracil, cisplatin, carboplatin, and/or paclitaxel. Patients were excluded if they were found to have systemic metastatic disease at the time of the operation, had a tumor that penetrated into an adjacent organ (T4), or had an incomplete pathologic resection (R1). In addition, patients who had an operation other than an en bloc or transhiatal esophagectomy were excluded. This study was approved by the Institutional Review Board of the University of Southern California.

En bloc esophagectomy was performed in patients who were younger than 75 years and who were free of substantial medical comorbidities. Transhiatal esophagectomy was performed in patients 75 years or older and those with significant cardiac or pulmonary disease or other major comorbid conditions. Patients were followed by the operating surgeon at regularly scheduled intervals (every 3 months for the first 3 years, every 6 months up to 5 years, and yearly thereafter). CT scans of the chest and abdomen and routine blood chemistry results were obtained at each visit, and PET scans were obtained yearly.

\section{Surgical Treatment}

En bloc esophagectomy was performed as described in previous publications. ${ }^{4}$ In brief, the en bloc dissection was performed through a right seventh-interspace posterolateral thoracotomy to have excellent exposure of the potentially involved mediastinal nodes below the carina, an upper midline laparotomy, and a left neck incision. The thoracic dissection removed en bloc the esophagus, azygos vein, thoracic duct, and surrounding lymph node-bearing mediastinal tissues. The borders of the dissection extend superiorly to above the azygos arch; laterally to include the left and right mediastinal parietal pleura; anteriorly to the membranous trachea, pericardium, and diaphragm; posteriorly to the spine and aorta; and inferiorly to the esophageal hiatus and down into the costal vertebral angle. Above the aortic arch, the dissection is kept adjacent to the esophagus to minimize potential injury to the recurrent laryngeal nerve. A paratracheal node dissection is not routinely performed for adenocarcinoma of the distal esophagus or gastroesophageal junction. The abdominal dissection was performed through an upper midline incision and removed the node-bearing tissue from the lateral surface of the right crus, the porta hepatis, and around the portal vein and the common hepatic, celiac, and left gastric arteries. Additionally, node-bearing tissue was removed from the lateral surface of the left crus, around the splenic artery, medial to the splenic hilum, and anterior to the adrenal gland. The en bloc dissection allowed systematic removal of lymph nodes in the following areas: low paratracheal, subcarinal, perihilar, paraesophageal, parahiatal, costalvertebral space, porta hepatis, superior retropancreatic, and around the portal vein and the hepatic, celiac, and splenic arteries.

Transhiatal dissection was performed as described by Orringer ${ }^{5}$ through an upper midline incision and a left neck incision. The lower mediastinal and esophageal dissection was performed through a widened diaphragmatic hiatus by using blunt and sharp dissection. Paraesophageal and mediastinal lymph nodes were removed as exposure allowed, but a complete node dissection in the mediastinum was not possible. The abdominal node dissection was identical to that described for the abdominal portion of the en bloc procedure.

Reconstruction in most patients was with a tubularized gastric pull-up, but if the stomach was unavailable or unsuitable, a colon interposition was performed. Reconstructions were preferentially placed in the posterior mediastinum, and the proximal anastomosis was hand sewn in the neck.

\section{Statistical Analysis}

Grouped data were expressed as medians with interquartile ranges. Statistical analysis appropriate for nonparametric data was used. Univariate analyses were performed by using the Fisher exact test for categorical variables and the Mann-Whitney test for continuous variables. Survival was calculated by using the Kaplan-Meier method, with comparisons of survival by means of the log-rank test.

\section{Results}

From January 1993 through August 2005, 416 patients with esophageal adenocarcinoma were seen by members of the 
TABLE 1. Characteristics of the study population

\begin{tabular}{|c|c|c|c|}
\hline Characteristic & $\begin{array}{c}\text { Transhiatal } \\
\text { esophagectomy } \\
\text { group }(n=18)\end{array}$ & $\begin{array}{c}\text { En bloc } \\
\text { esophagectomy } \\
\text { group }(n=40)\end{array}$ & $\begin{array}{c}P \\
\text { value }\end{array}$ \\
\hline Sex, F/M & $2 / 16$ & $5 / 35$ & 1.0 \\
\hline Age, y & 64 (59-74.5) & $54.5(49-61)$ & .0009 \\
\hline BMI & 27.05 (24.95-29.8) & $27.3(24.6-29)$ & .76 \\
\hline ASA score & 3 & 3 & .437 \\
\hline \multicolumn{4}{|l|}{ Comorbidity } \\
\hline Any & $15(83 \%)$ & $11(27.5 \%)$ & .0001 \\
\hline - Diabetes & 1 & 2 & \\
\hline - Hypertension & 5 & 6 & \\
\hline - Cardiovascular & 10 & 4 & \\
\hline - Pulmonary & 4 & 2 & \\
\hline \multicolumn{4}{|l|}{ Tumor location* } \\
\hline $\begin{array}{l}\text { Distal } \\
\quad \text { esophagus/GEJ }\end{array}$ & $88 \% / 12 \%$ & $90 \% / 10 \%$ & 1.0 \\
\hline $\begin{array}{l}\text { Presence of } \\
\text { Barrett's } \\
\text { esophagus }\end{array}$ & $22 \%$ & $28 \%$ & .76 \\
\hline Chemotherapy only & $2 / 18(11.1 \%)$ & $2 / 40(5 \%)$ & .58 \\
\hline Chemoradiotherapy & $16 / 18(88.9 \%)$ & $38 / 40(95 \%)$ & \\
\hline Radiation dose, Gy & $40(35.5-40)$ & $40(40-45)$ & .14 \\
\hline
\end{tabular}

All values are presented as medians and interquartile ranges. $B M I$, Body mass index; $A S A$, American Society of Anesthesiologists; GEJ, gastroesophageal junction. *Information allowing determination of a distal esophageal versus a gastroesophageal junction tumor was available on 9 patients undergoing transhiatal resection and 20 patients undergoing en bloc resection.

Thoracic and Foregut Surgery Division at the University of Southern California. From this group, 323 (78\%) patients underwent primary surgical resection, and 92 (22\%) patients had neoadjuvant therapy before resection. Among patients who had neoadjuvant therapy, 6 presented late for salvage resection with known recurrent disease, 14 underwent a procedure other than an en bloc or transhiatal resection (7 minimally invasive and 6 Ivor-Lewis or non-en bloc transthoracic resections), 7 were found to have metastatic disease at the time of operation, 3 had an incomplete (R1) resection, and 3 patients died perioperatively ( 2 en bloc and 1 transhiatal). These patients were excluded. Therefore the final study population was composed of 58 patients: an en bloc resection was performed in 40 patients, and a transhiatal resection was performed in 18 patients. Reconstruction was with a gastric pull-up in $93 \%$ of patients, with colon interposition performed after en bloc resection in 3 patients and after transhiatal resection in 1 patient. Clinical data are summarized in Table 1. The median radiation dose was $40 \mathrm{~Gy}$ in each group, and approximately $90 \%$ of patients in each group had both chemotherapy and radiotherapy. Furthermore, chemotherapy was standard cisplatin and 5-fluorouracil in 93\% of patients undergoing en bloc resection and $83 \%$ of patients undergoing transhiatal resection $(P=.36)$.
Complete pathologic response occurred in 17 (29.3\%) of 58 patients and was more common in the transhiatal group. Details of the final pathology are shown in Table 2. The final pathologic stage was similar in both groups, but stage 3 tumors tended to be more prevalent in the en bloc group (37.5\% for en bloc resection vs $28 \%$ for transhiatal resection, $P=$.6). The majority of patients in both groups had residual cancer in the resected esophagus. Furthermore, residual cancer was found in both the esophagus and at least 1 lymph node in $67 \%$ of patients. In those with no residual cancer in the esophagus $(\mathrm{n}=19)$, only $2(10.5 \%)$ patients had 1 or more involved lymph nodes.

Median follow-up tended to be longer in patients who had an en bloc resection (en bloc, 34.1 months; transhiatal, 18.3 months; $P=.18$ ). Recurrence of any type occurred in 18 (45\%) patients who had an en bloc resection compared with $13(68 \%)$ patients who had a transhiatal resection $(P=.087)$ at a median of 8.5 months. The recurrence was systemic in the majority of patients (Table 3 ). The prevalence of local-regional recurrence was significantly reduced in patients who had an en bloc resection.

Overall survival at 5 years was significantly better in patients who had an en bloc compared with a transhiatal esophagectomy after neoadjuvant therapy $(51 \%$ vs $22 \%$, respectively; Figure 1). There were 4 non-cancer-related deaths during follow-up: 3 in the en bloc group and 1 in the transhiatal group. If survival is compared by using only cancer-related deaths, the improvement with the en bloc resection is even more significant $(56 \%$ for en bloc vs $25 \%$ for transhiatal at 5 years, $P=.03$ ). In patients with a complete pathologic response, there was a trend toward improved 5-year survival with an en bloc resection compared with that after a transhiatal resection (en bloc, $70 \%$; transhiatal, $43 \%$; $P=.3$; Figure 2). Survival in patients with residual disease was significantly better after an en bloc resection compared with that after transhiatal resection (48\% vs 9\%, respectively; $P=.02$; Figure 3). Among the 30 patients with residual disease after neoadjuvant therapy who had an en bloc resection, there were 17 patients operated on more than 5 years ago, and from this group, 6 were alive, for an absolute 5 -year survival of $35 \%$. In contrast, 8 patients had a transhiatal esophagectomy after neoadjuvant therapy more than 5 years ago, and no patient survived for 5 years (longest survival, 38.5 months).

\section{Discussion}

The treatment of locally advanced esophageal adenocarcinoma is difficult, and survival is often poor in these patients from either local-regional failure, development of systemic disease, or both. In an effort to improve survival, chemoradiotherapy has been given before surgical resection, and although many phase 2 studies suggested a benefit with this approach, the results from randomized prospective trials, as well as a large-population based study, have been disappointing. ${ }^{2,6}$ 
TABLE 2. Postoperative pathologic findings

\begin{tabular}{|c|c|c|c|}
\hline Characteristics & $\begin{array}{c}\text { Transhiatal esophagectomy } \\
\text { group }(\mathbf{n}=\mathbf{1 8})\end{array}$ & $\begin{array}{l}\text { En bloc esophagectomy } \\
\text { group }(n=40)\end{array}$ & $P$ value \\
\hline Stage 0 & 7 & 10 & \\
\hline Stage I & 3 & 3 & \\
\hline Stage IIA & 1 & 8 & \\
\hline Stage IIB & 2 & 4 & \\
\hline Stage III & 5 & 15 & \\
\hline Complete pathologic response & $7 / 18(39 \%)$ & $10 / 40(25 \%)$ & .35 \\
\hline Residual disease in the esophagus (\%) & $10 / 18(56 \%)$ & $29 / 40(73 \%)$ & .23 \\
\hline Residual disease (esophagus or nodes) & $11 / 18(61 \%)$ & $30 / 40(75 \%)$ & .35 \\
\hline Residual tumor size, $\mathrm{cm}$ & $1.75(0-5.5)$ & $3(0-5)$ & .56 \\
\hline Nodes removed & $19(13.5-29)$ & $29.5(24.5-44.5)$ & .0016 \\
\hline Patients with at least 1 involved node $(\%)$ & $7 / 18(38.8 \%)$ & $19 / 40(47.5 \%)$ & .58 \\
\hline \multicolumn{4}{|l|}{ Involved nodes } \\
\hline Median & $0(0-4)$ & $0(0-3)$ & .64 \\
\hline Mean & 3.5 & 3.2 & \\
\hline \multicolumn{4}{|l|}{ Distribution of involved nodes (\%) } \\
\hline Abdominal and mediastinal & $2 / 7(28.5 \%)$ & $9 / 19(47.4 \%)$ & \\
\hline Mediastinal only & 0 & $1 / 19(5.2 \%)$ & \\
\hline Abdominal only & $5 / 7(71.4 \%)$ & $9 / 19(47.4 \%)$ & \\
\hline
\end{tabular}

Values are presented as medians and interquartile ranges.

The only randomized trial to show improved survival with preoperative chemoradiotherapy was published by Walsh and colleagues, ${ }^{7}$ but this trial has been dismissed by many because of the lack of appropriate staging, methodological flaws, and most importantly survival curves that do not match the data in the text. ${ }^{2,7,8}$ Trials from England have suggested a benefit from preoperative chemotherapy alone, but these trials also suffer from methodological flaws, and similar results were not found in a major US trial. ${ }^{9,10}$ The most encouraging results of these trials has been that patients that have a complete pathologic response appear to have improved survival, with most series reporting about 50\% five-year survival in this subgroup. ${ }^{11,12}$ Because overall survival is similar to that of patients treated with resection alone, this would imply that patients who do not have a complete pathologic response to therapy have worse survival than similarly staged patients treated with primary esophagectomy, and this has been substantiated in a number of reports. ${ }^{6,13}$ The survival after resec- tion in patients with residual disease after neoadjuvant therapy has been so poor that some oncologists and surgeons question the role of esophagectomy in this group.

We and others have reported excellent survival in patients with esophageal adenocarcinoma treated with primary en bloc resection, and in similarly staged patients with limited lymph node involvement, we have shown that survival was improved with the en bloc compared with the transhiatal esophagectomy. ${ }^{14-18}$ However, criticism regarding stage migration and patient selection, as well as the complexity of the en bloc operation, has hampered widespread acceptance of this procedure. The aim of this study was to compare the effect of the type of resection in a group of patients with locally advanced esophageal adenocarcinoma all treated with neoadjuvant therapy. Similar to our experience with primary resection, we found that overall survival after neoadjuvant therapy was significantly better in patients who had an en bloc compared with a transhiatal esophagectomy. Our $22 \%$ five-year

TABLE 3. Recurrence pattern after esophagectomy related to operative approach

\begin{tabular}{|c|c|c|c|c|c|c|c|}
\hline & \multicolumn{3}{|c|}{$\begin{array}{c}\text { Transhiatal esophagectomy } \\
\text { group (n=18) }\end{array}$} & \multicolumn{3}{|c|}{$\begin{array}{c}\text { En bloc } \\
\text { esophagectomy group }(n=40)\end{array}$} & \multirow[b]{2}{*}{$P$ value ${ }^{*}$} \\
\hline & CPR & RD & Total & cPR & RD & Total & \\
\hline Any recurrence (\%) & 3 & 10 & $13(68 \%)$ & 2 & 17 & $19(47.5 \%)$ & .095 \\
\hline Systemic & 2 & 7 & $9(50 \%)$ & 2 & 16 & $18(45 \%)$ & .78 \\
\hline Local-regional & 0 & 3 & $3(16.6 \%)$ & 0 & 0 & 0 & .023 \\
\hline Neck & 1 & 0 & $1(5.5 \%)$ & 0 & 1 & $1(2.5 \%)$ & .51 \\
\hline
\end{tabular}

$c P R$, Complete pathologic response; $R D$, residual disease. ${ }^{*}$ The $P$ value was calculated for the total number of recurrences between groups. 


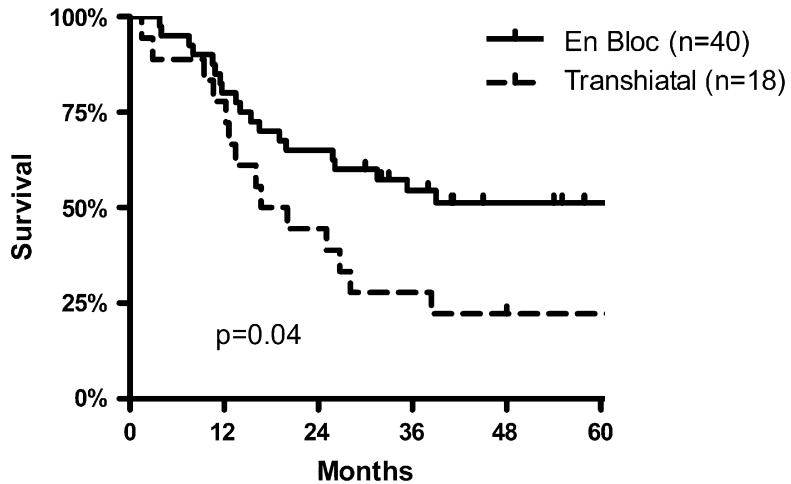

\begin{tabular}{l|ccccc} 
& $12 \mathrm{mos}$ & $24 \mathrm{mos}$ & $36 \mathrm{mos}$ & $48 \mathrm{mos}$ & $60 \mathrm{mos}$ \\
\hline En Bloc & 33 & 26 & 19 & 13 & 10 \\
THE & 14 & 8 & 5 & 4 & 3
\end{tabular}

Figure 1. Kaplan-Meier survival curve for all patients and allcause mortality. Overall survival is significantly better in the patients who had an en bloc resection compared with those who had a transhiatal resection after neoadjuvant therapy. Note that the 5 -year survival of $22 \%$ for the patients who had a transhiatal resection is similar to that seen in previous reports. ${ }^{11}$ Absolute 5-year survival was $11(50 \%)$ of 22 for patients who had an en bloc resection compared with $3(23 \%)$ of 13 for patients who had a transhiatal resection $(P=.28)$. THE, Transhiatal esophagectomy.

survival with transhiatal resection after neoadjuvant therapy is similar to what has been reported by others. ${ }^{6,11}$ This similarity confirms that although our transhiatal group of 18 patients is small, there is no reason to believe that larger numbers would substantially alter our results, and that the significant difference we found in favor of the en bloc resection is valid. The few non-cancer-related deaths in this population of patients indicate that regardless of comorbidities, the most likely cause of death in patients treated for locally advanced esophageal adenocarcinoma is recurrent cancer, and even after neoadjuvant therapy, the extent of resection is still an important determinant of ultimate survival from this disease.

The improved survival with an en bloc resection is likely due to the high frequency of involved lymph nodes both in the mediastinum and abdomen after neoadjuvant therapy and the fact that a systematic lymphadenectomy allows removal of both known and unknown (micrometastatic) disease. This is also the likely explanation for why survival steadily improves with an increasing number of lymph nodes removed in patients with esophageal cancer (data not shown). This same correlation for increasing survival with an increasing number of resected nodes has previously been shown to be true for gastric cancer. ${ }^{19}$ Resection of potentially involved lymph nodes is important, even after neoadjuvant therapy, because Prenzel and colleagues ${ }^{20}$ have shown that neoadjuvant therapy does not reliably eliminate lymph node disease.

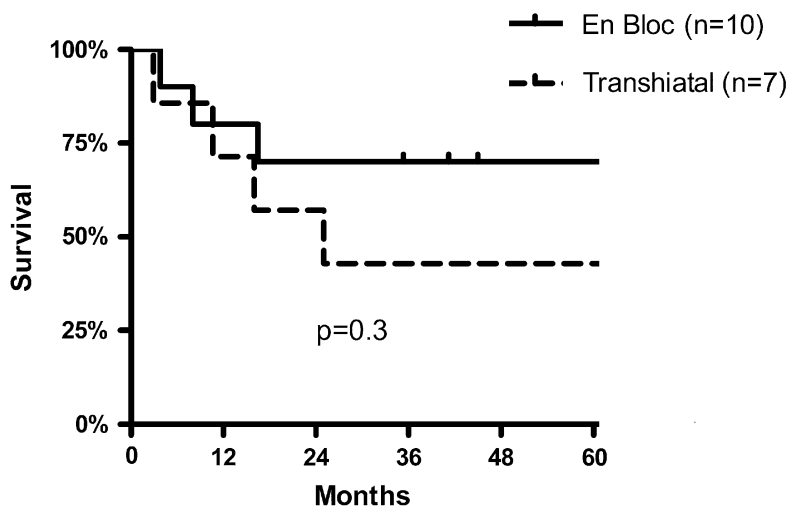

\begin{tabular}{l|ccccc} 
& 12 mos & 24 mos & 36 mos & 48 mos & 60 mos \\
\hline En Bloc & 8 & 7 & 6 & 4 & 4 \\
THE & 5 & 4 & 3 & 3 & 3
\end{tabular}

Figure 2. Kaplan-Meier survival curve for patients who had a complete pathologic response on final pathology. There was a nonsignificant improvement in survival for patients who had an en bloc resection. Note that the 5-year survival for patients who had a transhiatal resection and complete pathologic response was $43 \%$, and this again is similar to published results. ${ }^{11}$ Absolute 5-year survival was $5(100 \%)$ of 5 in the en bloc group versus $3(60 \%)$ of 5 in the transhiatal group $(P=.44)$. THE, Transhiatal esophagectomy.

These authors used both routine histology and immunohistochemistry to evaluate for micrometastatic nodal disease and reported that after neoadjuvant therapy, $75 \%$ of patients have nodal metastases. The high prevalence of residual nodal disease emphasizes the importance of complete node dissection and almost certainly explains our finding of improved survival with an en bloc resection, particularly because the incidence of systemic disease was similar in our en bloc and transhiatal resection groups.

Given the failure of randomized trials to demonstrate a clear survival advantage for all patients treated with neoadjuvant therapy before surgical resection, attention has focused on the subgroup of patients with complete pathologic response. We found that complete pathologic response on final pathology was present in $29 \%$ of patients in this retrospective analysis, and as has been previously demonstrated, these patients had improved survival compared with those with residual disease. The 5-year survival after complete response in patients who had a transhiatal resection was $43 \%$, which is similar to what has been reported in other series. ${ }^{11}$ However, the $70 \%$ five-year survival with an en bloc resection in patients with complete pathologic response sets a new high standard. It is interesting that nodal disease as the only form of residual disease (no tumor left in the esophagus) was rare. Given this, one would expect that in patients with complete pathologic response, the type of resection would not affect survival. However, we found a trend toward 


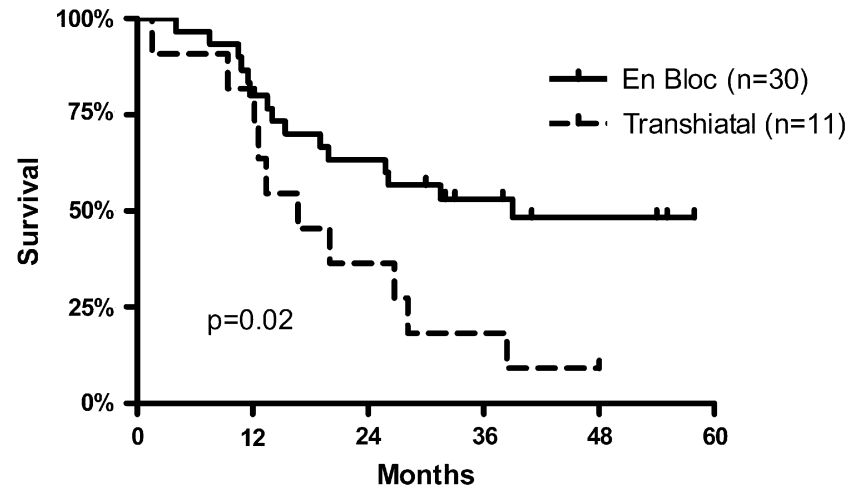

\begin{tabular}{l|ccccc} 
& 12 mos & 24 mos & 36 mos & 48 mos & 60 mos \\
\hline En Bloc & 24 & 19 & 13 & 9 & 6 \\
THE & 9 & 4 & 2 & 1 & 0
\end{tabular}

Figure 3. Kaplan-Meier survival curve for patients who had residual disease after neoadjuvant therapy on final pathology. Survival was significantly improved in the patients who had an en bloc resection compared with those who had a transhiatal resection. Absolute survival was $6(35 \%)$ of 17 with an en bloc resection compared with $0(0 \%)$ of 8 with a transhiatal resection $(P=.1)$. THE, Transhiatal esophagectomy.

improved survival in these patients after an en bloc resection, and with larger numbers, the difference might have been significant (type 2 error). It is likely that the en bloc resection is removing unknown micrometastatic nodal disease in these patients. This concept is supported by studies using immunohistochemistry to identify micrometastases in lymph nodes, such as one by Prenzel and colleagues,${ }^{20}$ in which $41 \%$ of patients thought to be node negative based on routine histology had micrometastases. This emphasizes the inaccuracy of only using routine histology to evaluate for complete pathologic response and reinforces the importance of a systematic lymphadenectomy to remove microscopic residual disease, prevent recurrence, and maximize survival, even in patients thought to be complete pathologic responders.

The most important finding in this study was that there was a significant improvement in survival with an en bloc resection compared with that with a transhiatal resection in patients with residual disease after neoadjuvant therapy and that the $48 \%$ five-year survival with en bloc esophagectomy justifies resection in these patients. Even when absolute survival is considered, we found that at 5 years, $35 \%$ of patients with residual disease were alive after an en bloc resection compared with 0 patients in the transhiatal group. The fact that these patients all had residual disease eliminates concerns regarding stage shift or differences in the extent of disease accounting for the improved survival in the en bloc group and confirms that the extent of resection directly affects the likelihood of survival from esophageal adenocarcinoma. The 9\% survival after transhiatal resection we observed in patients with residual disease is similar to the $12 \%$ survival reported for this group in the University of Michigan trial, and therefore it is not that our patients with residual disease who have a transhiatal resection are doing worse than expected. Rather, the clear message from our data is how well patients do longterm with an en bloc resection after neoadjuvant therapy, even in the setting of residual disease. These data should discourage the dismissal of the group with residual disease as incurable and encourage a systematic lymphadenectomy and complete resection to enable a chance for cure in these patients. However, in patients with residual disease, a transhiatal resection should not be routinely offered, given the poor survival with this operation in these patients.

The primary goal of surgical resection is complete disease removal in an effort to minimize local-regional recurrence, and we have previously shown that the en bloc technique is associated with a $1 \%$ local-regional failure rate ${ }^{14}$ In contrast, local-regional failure rates with transhiatal resection are $30 \%$ to $40 \%$ in series from around the world. ${ }^{11,21,22}$ The major effect of neoadjuvant therapy appears to be an improvement in local regional disease control without a reduction in systemic disease. In the randomized trial from the University of Michigan, local-regional disease recurrence was reduced from $42 \%$ in the transhiatal resection-only group to $19 \%$ in the neoadjuvant therapy group. ${ }^{11}$ The prevalence of localregional failure after neoadjuvant therapy and transhiatal resection in our experience was similar at $16.6 \%$. However, this is significantly higher than the complete absence of local regional failure we observed in patients who had an en bloc resection after neoadjuvant therapy. The similar low localregional failure rate with the en bloc esophagectomy, whether done as primary therapy or after neoadjuvant therapy, calls into question the utility of neoadjuvant therapy for local control unless a transhiatal operation is the only option for the patient. Similar to reports from the randomized trials of neoadjuvant therapy, we noted that the majority of recurrences were systemic, despite the administration of neoadjuvant therapy, and this frustrating fact represents a clear challenge for the oncology community and should encourage adoption of new approaches for this daunting disease, including consideration of long-term, low-dose adjuvant chemotherapy administration and chemosensitivity-directed chemotherapy protocols.

The shortcomings of this study include that it is retrospective and that the selection of patients for a transhiatal resection was based on advanced age and medical comorbidities. However, several facts show these shortcomings to be of minor significance. First, the survival and local-regional recurrence rates we report for the patients undergoing transhiatal resection are very similar to the results from randomized trials and from a large population-based database. ${ }^{6,11,12}$ Furthermore, the major cause of death in these patients was cancer, with only a single death in the transhiatal group occurring 
as a result of a non-cancer-related cause. Thus concerns regarding the significantly increased age and comorbidities in the transhiatal group affecting survival are unfounded, and an analysis of only cancer-related deaths merely strengthens the significance of our findings. Another issue is that we deliberately did not provide preoperative staging information because clinical staging for esophageal cancer is poor. However, our bias is to perform primary en bloc resection in patients with limited local-regional disease. Patients with locally advanced tumors with multiple nodes on endoscopic ultrasonographic analysis or PET scanning are referred for neoadjuvant therapy, and the decision for an en bloc versus transhiatal resection after neoadjuvant therapy is not based on response but is made purely on the basis of the age, comorbid conditions, and cardiopulmonary status of the patient. If anything, in this study the deck was stacked against the en bloc group, where follow-up was longest, fewer patients had a complete pathologic response, and $68 \%$ had stage 2 or 3 disease compared with $44 \%$ in the transhiatal group after therapy. Thus the survival differences we demonstrated are unlikely to be due to substantial differences in preoperative stage between the en bloc and transhiatal groups or as a result of the selection of patients with the best response to neoadjuvant therapy for an en bloc resection. Lastly, despite the differences between groups in regard to age and comorbidities, we found no evidence that the neoadjuvant therapy differed significantly between groups, with the majority of patients receiving standard chemotherapy and radiotherapy.

In conclusion, in this study of patients with locally advanced adenocarcinoma of the esophagus, all of whom were treated with neoadjuvant therapy, there was a significant improvement in overall survival and survival with residual disease in patients who had an en bloc esophagectomy compared with those who had a transhiatal resection. Clearly, even after neoadjuvant therapy, the extent of resection is an important determinant of long-term survival from esophageal adenocarcinoma. The explanation for this finding relates to the higher local-regional failure rate with a transhiatal resection and likely to the removal of both known and unknown (micrometastatic) disease with the extended lymphadenectomy that is performed with the en bloc procedure. Poor survival with a transhiatal resection in patients with residual disease mandates an en bloc resection in this group, and the improved survival overall should make an en bloc esophagectomy the procedure of choice in all patients after neoadjuvant therapy. Further improvements in survival with this deadly disease will require chemotherapy agents and protocols that are able to reduce the incidence of systemic recurrence and represent a major challenge for the oncology community. However, these compelling data proving the superiority of the en bloc resection in patients who have had neoadjuvant therapy should finally put to rest the question of whether an extended resection is necessary, beneficial, or both for the treatment of patients with adenocarcinoma of the esophagus.

\section{References}

1. Pohl H, Welch HG. The role of overdiagnosis and reclassification in the marked increase of esophageal adenocarcinoma incidence. $J$ Natl Cancer Inst. 2005;97:142-6.

2. DeMeester SR. Adenocarcinoma of the esophagus and cardia: a review of the disease and its treatment. Ann Surg Oncol. 2006;13:12-30.

3. Hagen JA, DeMeester SR, Peters JH, et al. Curative resection for esophageal adenocarcinoma: analysis of 100 en bloc esophagectomies. Ann Surg. 2001;234:520-31

4. Hagen JA, Peters JH, DeMeester TR. Superiority of extended en bloc esophagogastrectomy for carcinoma of the lower esophagus and cardia J Thorac Cardiovasc Surg. 1993;106:850-9.

5. Orringer MB. Transhiatal esophagectomy without thoracotomy for carcinoma of the thoracic esophagus. Ann Surg. 1984;200:282-8.

6. Rouvelas I, Zeng W, Lindblad M, Viklund P, Ye W, Lagergren J. Survival after neoadjuvant therapy compared with surgery alone for resectable esophageal cancer in a population-based study. World J Surg. 2006; 30:2182-90

7. Walsh TN, Noonan N, Hollywood D, et al. A comparison of multimodal therapy and surgery for esophageal adenocarcinoma [erratum appears in N Engl J Med. 1999;341:384]. N Engl J Med. 1996;335:462-7.

8. Walsh T. Correction to "A comparison of multimodal therapy and surgery for esophageal adenocarcinoma." N Engl J Med. 1999;341:384.

9. Group MRCMUGTC. Medical Research Council (MRC) randomised phase III trial of surgery with or without pre-operative chemotherapy in resectable cancer of the oesophagus [abstract]. Br J Cancer. 2000; 83(suppl 1): 1

10. Kelsen DP, Ginsberg R, Pajak TF, et al. Chemotherapy followed by surgery compared with surgery alone for localized esophageal cancer. N Engl J Med. 1998;339:1979-84.

11. Urba S, Orringer M, Turisi A, et al. Randomized trial of preoperative chemoradiation versus surgery alone in patients with locoregional esophageal carcinoma. J Clin Oncol. 2001;19:305-13.

12. Reynolds JV, Muldoon C, Hollywood D, et al. Long-term outcomes following neoadjuvant chemoradiotherapy for esophageal cancer. Ann Surg. 2007;245:707-16

13. Rice TW, Blackstone EH, Adelstein DJ, et al. Role of clinically determined depth of tumor invasion in the treatment of esophageal carcinoma. J Thorac Cardiovasc Surg. 2003;125:1091-102.

14. Hagen J, DeMeester S, Peters J, et al. Curative resection for esophageal adenocarcinoma: analysis of 100 en bloc esophagectomies. Ann Surg. 2001;234:520-31

15. Portale G, Hagen JA, Peters JH, et al. Modern 5-year survival of resectable esophageal adenocarcinoma: single institution experience with 263 patients. J Am Coll Surg. 2006;202:588-98.

16. Altorki NK, Girardi L, Skinner DB. En bloc esophagectomy improves survival for stage III esophageal cancer. J Thorac Cardiovasc Surg. 1997; 114:948-56.

17. Lerut T, Coosemans W, Decker G, et al. Extended surgery for cancer of the esophagus and gastroesophageal junction. J Surg Res. 2004;117:58-63.

18. Johansson J, DeMeester TR, Hagen JA, et al. En bloc vs transhiatal esophagectomy for stage T3 N1 adenocarcinoma of the distal esophagus. Arch Surg. 2004;139:627-33.

19. Smith DD, Schwarz RR, Schwarz RE. Impact of total lymph node count on staging and survival after gastrectomy for gastric cancer: data from a large US-population database. J Clin Oncol. 2005;23:7114-24.

20. Prenzel K, Konig A, Schneider P. Reduced incidence of nodal micrometastases after major response to neoadjuvant chemoradiation in locally advanced esophageal cancer. Ann Surg Oncol. 2007;14:954-9.

21. Hulscher JB, van Sandick JW, Tijssen JG, et al. The recurrence pattern of esophageal carcinoma after transhiatal resection. J Am Coll Surg. 2000;191:143-8.

22. van Sandick JW, van Lanschot JJB, Ten Kate FJ, et al. Indicators of prognosis after transhiatal esophageal resection without thoracotomy for cancer. J Am Coll Surg. 2002;194:28-36.

\section{Discussion}

Dr Richard I. Whyte (Stanford, Calif). First, I would like to congratulate Dr Rizzetto on a very interesting and well-presented paper 
that really adds to our knowledge on esophageal cancer, the role of en bloc esophagectomy, and the results of neoadjuvant therapy.

The authors are clearly presenting some very impressive results here in terms of a $70 \%$ five-year survival in the $25 \%$ of patients who were complete pathologic responders. That is really sort of best in class. Also, they really demonstrate the fact that en bloc resection results in a very low local recurrence rate.

I have 2 or 3 questions, however, and I think Dr DeMeester is going to address those.

First, you have described candidates for neoadjuvant therapy as having locally advanced disease proved by both endoscopic ultrasonographic analysis and PET scans, yet there are no data in the presentation or in the manuscript on the TNM staging of these patients. Can you provide some information as to their pretreatment TNM status?

Dr DeMeester. The problem with pretreatment staging is that the clinical staging for esophageal cancer is terrible. Many of these patients in the earlier time frame did not undergo PET scans or endoscopic ultrasonography. We do that routinely now, and as you know, our preference is primary surgical resection in anyone who we think has limited local regional disease, which for us, is patients who had any depth of tumor with less than 5 obvious nodes determined by means of endoscopic ultrasonographic analysis or PET scan. For anyone with 5 or more nodes, we know that the systemic failure rate with surgical intervention alone in our experience exceeds $80 \%$, and therefore those patients will need chemotherapy either before or after surgical intervention. We talk to the patient about that, and many of these patients are selected then to have preoperative chemoradiotherapy, followed by surgical intervention. All of our patients who have neoadjuvant therapy have extensive local-regional disease. In fact, if you look at the final pathologic staging, some $60 \%$ of the en bloc group had stage II or III disease after neoadjuvant therapy, and therefore the majority of these patients started with significant disease.

Dr Whyte. My next question sort of relates to how patients were assigned to receive an en bloc resection or a transhiatal resection. In the presentation we heard that there is use of the number of lymph nodes to sort of ascribe one operation versus another. However, in the manuscript you talk about it both being related to the age and the presence or absence of comorbidities. I am a little confused then as to whether you used the presence or absence of lymph nodes or the number of lymph nodes to help determine whether patients receive an en bloc resection or a transhiatal resection.

Dr DeMeester. I am sorry if that was confusing in the presentation. We are merely demonstrating that, as should be no surprise, en bloc resection removes far more lymph nodes than transhiatal resection, but the number of lymph nodes has nothing to do with the selection of the operation. The operation is selected on our assessment of the patient's physiologic ability to withstand an en bloc procedure, which is a bigger deal, and therefore patients who are older than 70 or 75 years and have any renal, cardiac, or pulmonary dysfunction are selected for a transhiatal resection. That then brings in the concern that patients in the transhiatal group have higher comorbidities and are dying off as a consequence of these comorbidities and not their cancer. We also prepared cancer-only survival statistics and have demonstrated that the statistics and the significance between the curves are the same. In fact, all deaths, except for one in the transhiatal group, were from cancer. Therefore the comorbid- ities are what we use to select the procedures, but that had no effect on the outcome from the procedures.

Dr Whyte. So you are not using the presence or absence of nodal disease or the number of nodes to assign someone to en bloc versus transhiatal resection.

Dr DeMeester. No. We use the number of nodes to determine whether we should select patients for neoadjuvant therapy, but that has nothing to do with whether we select them for transhiatal or en bloc resection. Our preference is always en bloc resection, but we will downstep to a transhiatal resection in patients who we fear will not handle the en bloc resection based on comorbidities.

Dr Whyte. My last question then is this: Why don't you do an en bloc resection on everyone? Your mortalities are pretty similar. In terms of the ASA class you presented, they are roughly the same in the transhiatal versus the en bloc resection. As for the presence or absence of comorbidities, the numbers are fairly similar. You had 11 of 40 patients who had significant comorbidities in your en bloc resection group. I am thinking that persons who undergo a transhiatal resection have a laparotomy, a gastric mobilization, a pretty extensive mediastinal dissection, and then a neck incision, and the patients undergoing en bloc resection get a bit more extensive abdominal dissection and then perhaps a rather limited thoracotomy but, in addition, a much more precise mediastinal dissection. I am wondering why you do not do an en bloc esophagectomy in everyone, particularly because you are clearly convinced it is the right operation. Your data indicate that they have better long-term survival, and the mortalities are somewhat similar. Why not do an en bloc resection on everyone? Then we can really see-sort of take out this transhiatal versus en bloc issue - and focus on whether the potential increased morbidity of the bigger operation is more than made up for by the better long-term survival in these patients.

I really enjoyed the paper, and it was well presented. Thanks very much.

Dr DeMeester. We are pretty much coming to that same conclusion. Essentially everyone who comes for primary surgical resection has an en bloc resection at this point. I have done 1 transhiatal resection maybe in the last 3 years, and that was in a guy who had a previous pneumonectomy. Therefore essentially everyone gets an en bloc resection for primary therapy. Part of this experience now is teaching us that even after neoadjuvant therapy, we need to push the en bloc resection because part of our attitude initially, as I think was present in many surgeons, was that if you had neoadjuvant therapy, that was supposed to take care of the problem, and you could probably just get by with a transhiatal resection. Therefore 5 or 10 years ago, when we were first doing this, often we would do a transhiatal resection in a patient who could have had an en bloc resection, but we thought they have had neoadjuvant therapy, the chemoradiation increases the potential morbidity particularly for a thoracotomy, more respiratory complications, maybe it is not worth doing that. These data now will tell us that anybody who comes with neoadjuvant therapy at USC will get an en bloc resection if at all possible.

Dr Robert Cerfolio (Birmingham, Ala). Steve, excellent work. I congratulate you and your efforts to continue to collect prospective data on esophageal cancer. It is a lot of work, and we appreciate everything you have contributed to the field and to our patients.

This thing about complete responders-the only way to know who is a complete responder is to take the cancer out and look. 
We have generated some data on 170 patients now-we prefer IvorLewis esophagogastrectomy, as you do-and have shown that the change in the maximal standardized uptake value helps predict who will be a complete responder and who will not. When the primary tumor decreases by greater than $75 \%$, that patient is very likely-has a greater than $90 \%$ chance of being a complete responder. I am wondering whether you have any data that would corroborate that, and then, if you do, if you would comment on the fact that I get a lot of medical oncologists who say, "If he is a complete responder, he does not need surgery." The ones who refuse or decline it because they are a "complete responder" all seem to return within 9 to 12 months with bad local and often distant metastatic disease. Can you tell me how you respond to your oncologists and push the patient to get the operation and if you have maximal standardized uptake value data?

Dr DeMeester. We are just starting to look at some of the maximal standardized uptake value data. We do not certainly have that going back in this study population, particularly because a number of these patients had their operations before PET scans were widely used. I will say, though, that I am not convinced that PET scanning is ever going to get down to the level of the cell. There is just no chance that you are going to predict $100 \%$ of the time who has no residual cancer. I do not think there is any test short of the pathologist with his microscope sorting that out. The reality is, if you leave cancer behind, the patients are going to die from their cancer. It is going to come back. Therefore I do not see a way with any of our current studies to rule out or to find those patients who do not need surgical intervention because of complete pathologic response. We know that the mucosal disease clears up first. If you cannot clear mucosal disease with neoadjuvant therapy, then you have real problems. In our data $60 \%$ of patients had residual disease in the esophagus, and therefore the majority of patients do, and it should be resected with an en bloc procedure.

Dr Cerfolio. Therefore you tell the oncologists that you do not know whether the patients are complete responders no matter what their repeat endoscopic ultrasonographic tests and repeat PET scans show, and thus you, like us, recommend surgical resection.

Dr DeMeester. Repeat endoscopic ultrasonography has been shown to be of no value after neoadjuvant therapy, and therefore I do not even offer it. I tell the oncologists that $60 \%$ of these patients have residual disease in the esophagus and $40 \%$ will have nodal disease, and if you read a recent article by Prenzel using immunohistochemistry, $75 \%$ of patients after neoadjuvant therapy have either histologic or immunohistochemical evidence of nodal disease. The vast majority of these patients have disease and should have an esophagectomy. Now the issue you brought forward is that many oncologists have taken an approach that surgical intervention should not be offered after neoadjuvant therapy because if you get a complete pathologic response, your survival is pretty good, and if you did not and you get a transhiatal resection, your survival is horrible, and therefore why should they undergo an operation at all after neoadjuvant therapy? That is the emphasis of this paper. Your survival is not horrible if you do a good operation. Fifty percent of patients were alive at 5 years after neoadjuvant therapy with an en bloc resection, and even with residual disease, it was an impressive survival. These patients should have an esophagectomy, and it should be en bloc.
Dr Cerfolio. Our data are similar, and we agree.

Dr David Follette (Sacramento, Calif). Steven, I enjoyed your paper, and it is one of the first to corroborate some data from East Germany regarding complete responders and survival in squamous cell carcinoma of the esophagus, which we do not see that much of anymore. We really still do not know the benefit of neoadjuvant therapy because the Walsh study is one of the only ones to show the advantage and, as was mentioned in the talk, is severely flawed. In my mind, what is coming to the fore is this issue of a complete response or a partial response. We have seen good long-term survival in persons who are nonresponders to neoadjuvant therapy with good survival rates with just definitive chemotherapy and radiation therapy. My question is, you briefly mentioned to Rob that you do not do repeat ultrasonographic analysis on these persons, but do you use CT scanning restaging after neoadjuvant, and if they are nonresponders or if the tumor is fed and growing with the neoadjuvant, how do you handle that group of patients?

Dr DeMeester. We definitely restage them, looking for systemic disease. If a patient has systemic disease, we do not offer him or her esophagectomy. If the patient does not have systemic disease, the extent of the local regional disease does not dissuade me from moving forward. It is important to separate out squamous tumors from adenocarcinomas. All these were adenocarcinomas. You cannot really do an en bloc resection for squamous tumors. You have millimeters between the trachea and major vessels, and therefore it is a completely different disease, and for those patients, I think definitive chemoradiotherapy often is an advantage. It is totally different for a distal esophageal adenocarcinoma. Most Americans have centimeters of fat between any significant structure and the esophagus, and you can get a very excellent resection and take out the lymph nodes, and there is no comparison between the results of good operations and definitive chemoradiotherapy in patients with distal esophageal adenocarcinoma.

Dr Alex Little (Dayton, Ohio). I have one quick follow-up question to something that was put before. Is the difference in the operation because of the extent of the abdominal dissection or because there was not a thoracic nodal dissection?

Dr DeMeester. It is brought out in the manuscript, but our abdominal dissection is identical whether you have a transhiatal or an en bloc resection, and therefore it is the mediastinal dissection. As you saw, about $45 \%$ of patients had mediastinal and abdominal nodes in the en bloc group, and therefore there is a high prevalence of mediastinal nodes that I just do not think you can clear well, leading to the higher recurrence rate and the failure of survival in the transhiatal group.

Dr Little. If I might, I have one quick comment to ask you to react to, and it has already been alluded to. I think fully convincing evidence will not be put before us until all comers are randomized: to compare a relatively fit with a relatively unfit group sort of stacks the deck ahead of time.

Dr DeMeester. That would be true if they were dying from their comorbidities, but as I said, there was 1 death from comorbidities, a non-cancer-related death, in the transhiatal group. All the rest of the deaths were from cancer, and if we analyzed it based on cancer-related deaths only, the statistical significance is actually even greater. In my mind the issue is closed. It is decided. The argument is over. En bloc esophagectomy is superior to a transhiatal resection. 